\title{
Magnesio, un electrolito algo olvidado
}

\section{Magnesium, a somewhat forgotten electrolyte}

El magnesio $(\mathrm{Mg})$ es un catión divalente almacenado básicamente en el hueso y en los compartimentos intracelulares del músculo y los tejidos blandos; menos del 1\% del magnesio corporal total está en el extracelular principalmente en el plasma $(1,2)$. El balance de magnesio corporal depende del equilibrio entre la absorción intestinal y la excreción renal. Por ello, en un sujeto normal, la ingesta disminuida de magnesio se equilibra con una mayor absorción de magnesio en el intestino y una reducción de la excreción renal.

El 80\% de magnesio plasmático es filtrado por el glomérulo; $95 \%$ es reabsorbido. A diferencia de otros iones, el $60-70 \%$ de la reabsorción de magnesio ocurre en el asa gruesa de Henle; en el túbulo proximal se reabsorbe sólo 15-25\% del magnesio filtrado. El túbulo distal, es el sitio de control final de la excreción de magnesio, en él se re absorbe entre 5 y $10 \%$ del magnesio filtrado. En el asa gruesa de Henle, el magnesio es reabsorbido con el calcio de manera pasiva a través de la vía paracelular formada por uniones intercelulares estrechas (3).

El magnesio participa en numerosas reacciones enzimáticas, procesos de transporte, y síntesis de proteínas y ácidos nucleicos. Estabiliza las enzimas en muchas reacciones que generan ATP, antagoniza el calcio en la contracción muscular, modula la señal de transducción y proliferación celular de la insulina y es importante para la adhesión celular y el transporte de membrana. A pesar de su importancia fisiológica, su importancia clínica con frecuencia se subestima (2). De hecho, los trastornos del magnesio casi no se mencionan o se mencionan muy poco en la mayoría de los libros de Medicina; cuando lo mencionan se hace como parte de las alteraciones del calcio o del potasio.

Varios estudios epidemiológicos han demostrado asociación entre los niveles bajos de magnesio y aumento en el riesgo del síndrome metabólico, diabetes mellitus tipo 2, hipertensión y arterioesclerosis (4). Sin embargo, su uso como agente terapéutico solamente está indicado en la preeclampsia, para reducir el riesgo de eclampsia y en algunas formas específicas de arritmias: Torsade de pointes en pacientes con síndrome del QT largo y arritmias inducidas por digoxina (2). También se ha demostrado que el magnesio inhibe la formación de cálculos renales mediante la formación de complejos con oxalato y fosfato, además, la hipomagnesuria es un hallazgo común en formadores de cálculos (5).

En este número, se publica el estudio de Cieza JA et al (6), quienes encuentran hipomagnesemia en el 16,4\% e hipermagnesemia en el 18\% de pacientes adultos admitidos para observación intrahospitalaria u hospitalización en la sala de emergencia de un hospital general que atiende personas de bajos recursos económicos. Los investigadores encuentran asociación entre hipermagnesemia y nivel de urea sérica, y de hipomagnesemia con alcalosis metabólica y sepsis, de manera similar a lo encontrado en otros estudios; no se encontró relación de las alteraciones del Mg con la edad.

La hipomagnesemia se puede producir por disminución en la ingesta, redistribución del extracelular al intracelular, por pérdida gastrointestinal o por pérdida renal. La disminución en la ingesta rara vez causa hipomagnesemia, ya que la mayoría de alimentos contienen cantidades suficientes de magnesio y el riñón es capaz de adaptarse y conservar magnesio de manera muy eficiente. Sin embargo, puede ocurrir en pacientes desnutridos, alcohólicos y a quienes se les administra nutrición parenteral total durante tiempos prolongados (3).

A pesar que el $\mathrm{Mg}$ total permanece constante con la edad en sujetos sanos, el envejecimiento representa un factor de riesgo importante para la deficiencia de magnesio. Se ha observado reducción del magnesio libre intracelular con la edad. Los mecanismos serían: la inadecuada ingesta de nutrientes, posible reducción en la eficiencia de 
la absorción de $\mathrm{Mg}$ (asociada a niveles reducidos de vitamina D), excreción urinaria aumentada, enfermedades asociadas y comorbilidades, y uso de medicamentos como diuréticos (7).

Por otro lado, la hipermagnesemia, puede ocurrir en pacientes con enfermedad renal crónica (ERC) en estadios 4 ó 5, en especial en los no diabéticos. En ERC leve o moderada (estadios 1-3), se produce aumento en la excreción fraccional de magnesio, compensando la pérdida de la función renal de tal manera que los niveles de magnesio sérico se mantienen dentro de lo normal (2).

En los últimos 15 a 20 años, se han realizado estudios que han contribuido al conocimiento de la fisiología y los efectos de la suplementación de $\mathrm{Mg}$ (2), pero aún falta mucho por investigar. Como punto de partida, empecemos a medir de manera rutinaria la concentración sérica de magnesio en el paciente hospitalizado, en pacientes con hipokalemia o hipocalcemia, y en pacientes con alto riesgo de pérdida de magnesio. Asimismo, medir magnesio sérico antes de prescribir suplementación, porque podríamos generar hipermagnesemia y sus consecuencias. .

Juan Miyahira ${ }^{1, a}$

\section{REFERENCIAS BIBLIOGRÁFICAS}

1. Konrad M, Schlingmann KP, Gudermann T. Insights into the molecular nature of magnesium homeostasis. Am J Physiol Renal Physiol. 2004; 286: F599-F605.

2. de Francisco ALM, Rodríguez M. Magnesio y enfermedad renal crónica. Nefrología. 2013; 33(3):389-99. doi:10.3265/Nefrolog_a.pre2013. Feb. 11840

3. Pérez González E, Santos Rodríguez F, Coto García E. Homeostasis del magnesio. Etiopatogenia, clínica y tratamiento de la hipomagnesemia. A propósito de un caso. Nefrología 2009;29(6):518524.
4. Geiger H, Wanner C. Magnesium in disease. Clin Kidney J. 2012;5(Suppl 1): i25-i38.

5. Jara A, Bover J, Felsenfeld AJ, Nemeh M, Levine BS. Divalent ion metabolism. Current Nephrology. 1995; 18:133-182.

6. Cieza JA, Agurto CS, Gayoso DD. Evaluación y relevancia del magnesio sérico en pacientes adultos incidentes a la sala de emergencia de un hospital general del Ministerio de Salud Lima, Perú. Rev Med Hered. 2018; 29:69-75.

7. Barbagallo $M$, Belvedere $M$, Dominguez LJ. Magnesium homeostasis and aging. Magnesium Research. 2009; 22 (4): 235-46. 